Rev. Latinoam. Psicopat. Fund., São Paulo, 17(3-Suppl.), 666-672, set. 2014

http://dx.doi.org/10.1590/1415-4714.2014v17n3-Suppl.p666.8

\title{
Le lien social pervers
}

\author{
Christian Hoffmann*1
}

La définition lacanienne de la perversion comme saturation du manque dans l'Autre par l'objet pulsionnel est incompatible avec la philosophie néolibérale du lien social et sa définition d'un sujet autonome qui ne reconnaît pas l'Autre comme lieu d'une altérité dans un lien social tissé par la marchandisation du corps et des jouissances.

Mots clés: Perversions, Lacan, philosophie néolibérale

${ }^{* 1}$ Université Denis Diderot-Paris VII (Paris, França). 


\section{ARTIGOS}

Mon exposé est structuré en quatre parties, à savoir:

La structure lacanienne de la perversion

Le «discours pervers»

Le néolibéralisme

Peut-on parler d'un lien social — d'un discours — pervers dans une société néolibérale?

\section{La structure lacanienne de la perversion}

Dans son séminaire du 13 mai 1964 sur Les quatre concepts fondamentaux de la psychanalyse, Lacan nous fait remarquer que c'est le fantasme qui soutient le désir, ce n'est pas l'objet qui est le soutien du désir. Ce qui nous permet de comprendre la condition de "désirant" du sujet. Rêver de la perversion permet au névrosé de soutenir son désir, ça n'en fait pas un pervers. Lacan disait justement, «la perversion va au névrosé comme des guêtres à un lapin».

La structure perverse, comme le dit Lacan dans ce séminaire, est un effet inverse du fantasme, à savoir que le sujet pervers se détermine lui-même comme objet, et ceci dans sa rencontre avec la division subjective. Cliniquement, ça veut dire que dans cette rencontre subjective avec l'Autre, le sujet ne se divise pas, au contraire: il se fait l'objet pour la jouissance de l'Autre, sans le savoir.

La pulsion sado-masochiste se constitue lorsque le sujet se fait l'objet de la volonté de l'Autre au bénéfice de sa jouissance. L'article de Lacan Kant avec Sade illustre sur cette identification déniée du sujet à l'objet.

Dans son séminaire de 1968-69, D'un Autre à l'autre, Lacan consacre toute la séance du 26 mars 1969 à la «Clinique de la perversion». 
Lacan commence par définir l'incomplétude de l'Autre par l'évacuation de la jouissance de ce lieu par le fait que l'Autre maternel en tant que premier Autre de l'enfant est soumis à la loi de l'interdit de l'inceste, interdit qui décomplète l'amour maternel de l'érotique. C'est ce manque dans l'Autre qui définit l'objet $a$ de Lacan.

Le jeu pulsionnel entre l'objet $a$ et le manque dans l'Autre nous donne les coordonnées de la structure lacanienne des perversions. Lacan ne retient pas le «mépris pour l'autre» pour définir la perversion. Son point cardinal est que «le pervers se consacre à boucher le trou dans l'Autre». L'Autre existe ainsi pour le pervers qui devient un ardent «défenseur de la foi».

L'exhibitionniste cherche à faire apparaître le regard chez l'Autre, au-delà de la limite imposée à la jouissance par le principe de plaisir. Le résultat est la production de la jouissance au-delà de cette limite du refoulement dans l'Autre

Le voyeur force le regard au-delà du visible sur ce qui ne peut pas se voir. Ce n'est pas pour rien, nous rappelle Lacan, qu'une fente on l'appelle un regard. Le voyeur interroge le manque dans l'Autre et y suppléé par le regard. Contrairement à la névrose qui se définit par le mot d'ordre: «Circulez, il n'y a rien à voir», le pervers force le regard au-delà de la limite du refoulement, en donnant une consistance d'objet à l'Autre par une suppléance de son manque. Nous pouvons comprendre maintenant que le pervers croit à l'Autre jusqu'à se faire «l'auxiliaire de Dieu». Il suffit de lire Bataille.

Le masochiste fait surgir la voix de l'Autre à laquelle il va répondre avec fidélité comme un chien. Nous comprenons que la fonction du surmoi avec sa grosse voix et son impératif de jouissance est le ressort de cette perversion. Bref, le masochiste donne de la voix à l'Autre en le complétant de cet objet. Remarquons que la grosse voix du surmoi revient de l'Autre à travers ses impératifs de jouissance à la place de la voie symbolique forclose. Ce que nous préciserons à partir de la lecture de Sade par M. Foucault.

Le sadique à sa façon cherche à compléter l'Autre en lui dérobant la parole pour lui imposer sa voix. Lacan indique le ratage du sadisme dans cette opération d'annulation du symbolique en se référant à Sade, qui commente le moindre acte en l'incluant de surcroît dans un ordre. Ce que nous retrouverons également chez M. Foucault.

Lacan conclut sa «clinique de la perversion» en concluant sur la structure des pulsions déterminée par un «trou topologique».

La littérature est pourvoyeuse de perversions - nous verrons avec $\mathrm{M}$. Foucault le rapport entre la lettre et la perversion -, ouvrons la biographie de Alain Robbe-Grillet écrite par son épouse Catherine (Le livre porte simplement comme titre le prénom Alain). Elle dit de son mari qui rédigea un «Contrat» au début de leur relation que: «sa fantasmatique (celle d'Alain) tournait obsessionnellement autour d'une domination sadique sur de (très) jeunes femmes, à défaut de fillettes». Il n'est 
pas inutile d'indiquer que Lacan lui a déconseillé de faire une psychanalyse «comme s'il avait tout à y perdre» (commentaire de Catherine Robbe-Grillet). Le «Contrat de prostitution conjugale» commence ainsi: «Entre les soussignés, le présent contrat a été passé en vue de préciser les droits spéciaux du mari sur sa jeune épouse, lors de séances particulières, rétribuées en espèces, pendant lesquels la jeune femme subira des mauvais traitements, humiliations et tortures, dépassant les limites assignées aux exercices ordinaires, limites consacrées par l'habitude au cours de la première année du mariage». Le contrat stipule que «l'épouse se présentera (...) en attendant qu'on dispose d'elle (...) pour satisfaire les vices du mari (...) et jamais dans l'intention de lui faire éprouver une jouissance». Bref, une relation d'esclavage qui soumet l'autre à un objet sans parole. Seul est toléré l'imploration du maître à bien vouloir atténuer ses impératifs. Alain était un maître défaillant qui malgré son impuissance «restait le maître de et dans nos rites conjugaux».

\section{Le discours pervers}

Dans une conférence sur Sade en 1970, publiée dans La belle étrangère, M. Foucault pose l'existence d'un discours pervers. A travers une interrogation sur l'alternance chez Sade entre le discours et les scènes érotiques, il définit le discours comme le moteur d'un «désir illimité» dont la jouissance facilite le passage à l'acte qui répond à l'impératif sadien: «commettez ensuite».

M. Foucault reconnaît quatre types de discours: le discours de l'inconscient, le discours schizophrène, le discours idéologique ou philosophique ou religieux et le discours libertin ou pervers.

Le discours pervers nie tout ce qu'affirme le discours philosophique. Pour M. Foucault le discours philosophique joue essentiellement «un rôle castrateur». Dans l'Occident, depuis Platon, ce discours fonderait l'identité du sujet sur une renonciation d'une partie de lui-même, à savoir: le monde, le corps, le temps et le désir. Le discours de Sade vient en opposition à la philosophie avec une fonction de «décastration». Il s'agit non pas de dépasser la castration, mais «de nier, de dénier et de refuser la castration elle-même», par un jeu de négations de Dieu, de l'âme, de la loi et de la nature. Dieu n'existe pas. «Par conséquent la nature n'existe pas, la loi n'existe pas, l'âme n'existe pas et par conséquent tout est possible et rien n'est plus refusé dans l'ordre des prescription (...) donc je désire». Bref, le sujet n'aurait plus à sacrifier une partie de son narcissisme pour désirer sans limite.

M. Foucault donne un entretien en 1976 qui s'intitule Sade sergent du sexe. Il fait la remarque que chez Sade le corps est encore fortement organique. C'est l'organe qui est l'objet du sadisme: «Tu as un œil qui regarde, je te l'arrache». 
Par contre, le cinéma de ces années commence à «démanteler cette organicité», ce qui fait de Sade «un sergent du sexe» qui a formulé «l'érotisme propre à une société disciplinaire». Bref, le sadisme était «anatomiquement sage». Le monde contemporain voit défiler un «érotisme non disciplinaire: celui du corps».

\section{Le néolibéralisme}

G. de Lagasnerie développe clairement le paradigme néolibéral dans son ouvrage La dernière leçon de Michel Foucault. Sur le néolibéralisme, la théorie et la politique. La vision de la société néolibérale consiste à instituer une véritable marchandisation de la société. La loi serait celle du marché et elle engloberait l'ensemble des aspects de la vie en société, son action consiste à intervenir sur cette société pour que les mécanismes concurrentiels puissent jouer le rôle régulateur entre l'offre et la demande à chaque niveau du social. La subjectivité néolibérale définit «un homo oeconomicus qui ne renonce jamais à son intérêt, égoïste, sans transcendance». Ce sujet s'oppose à l'homo juridicus par son refus de renoncer à ses droits et de les transférer à un tiers souverain, garant de l'unité sociale. Il est pensé comme «un être unifié, cohérent et censé appliquer le calcul économique à toutes choses». Le «contrat» vient se substituer à la contrainte sociale.

Le geste critique de Foucault consiste à déplacer le concept majeur du néolibéralisme de «la liberté» vers «la pluralité».

\section{Peut-on parler d'un lien social pervers dans une société néolibérale?}

Pour aborder cette question, il faudrait revisiter le paradigme néolibéral avec les concepts lacaniens de sujet divisé, d'objet $a$, de l'Autre, du signifiant maitre, du surmoi, ce qui nous entrainerait dans un travail beaucoup trop long à exposer dans une seule conférence.

Par contre, je choisirai un autre angle, qui est celui que M. Foucault évoque à peine dans l'entretien dont le titre en dit long sur la fonction du surmoi dans la perversion. Dans Sade, sergent du sexe, il perçoit la différence entre «le corps chez Sade (qui) est encore fortement organique» et le corps contemporain formulant «un érotisme non disciplinaire».

Nelly Arcan s'est présentée comme philosophe et comme prostituée. Elle a décrit dans son dernier livre Burqa de chair ce qu'on peut appeler la marchandisation du corps: «Sur le Web, il fait froid. Le Web est un portail sur la 


\section{ARTIGOS}

désincarnation (...) Quand on peut voir son propre sexe ouvert devant soi et quand son sexe se met à parler, à renseigner, à étaler ses produits, à donner son prix et ses disponibilités, on franchit une ligne. Au-delà la folie guette, gueule ouverte, si grande et profonde qu'elle donne le vertige».

Reste à savoir si le démantèlement contemporain du corps organique est compatible avec l'objet $a$, avec lequel Lacan élabore la clinique de la perversion.

\section{Résumé}

\section{(O laço social perverso)}

A definição lacaniana de perversão como saturação da falta no Outro pelo objeto pulsional é incompativel com a filosofia neoliberal du laço social e sua definição de um sujeito autônomo que não reconhece o Outo como lugar de uma alteridade no laço social tecido pela mercantilização do corpo e dos gozos.

Palavras-chave: Perversão, Lacan, filosofia neoliberal

(Perverse social bonds)

Lacan's definition of perversion as saturation of the lack in the Other by the object is incompatible with the neoliberal philosophical approach of social bonds and its definition of an autonomous subject that fails to recognize the Other in a place of otherness in the social bond produced by the mercantilization of the body and of jouissance.

Key words: Perversion, Lacan, neoliberal philosophy

(El vínculo social perverso)

La definición lacaniana de la perversión como la saturación de la falta en el Otro por el objeto pulsional es incompatible con la filosofía neoliberal del lazo social y la definición de un sujeto autónomo que no reconoce al otro como un lugar de la alteridad en lazos sociales tejidos por la mercantilización de los cuerpos y goces.

Palabras clave: Perversiones, Lacan, filosofía neoliberal

(Die perverse soziale Bindung)

Die lacansche Definition von Perversion als Sättigung des Mangels im Anderen durch das Triebobjekt ist nicht kompatibel mit der neoliberalen Philosophie der sozialen Bindung und ihrer Definition eines autonomen Subjektes. Dieses Subjekt erkennt den Anderen nicht als Ort einer Alterität im sozialen Gefüge an, das durch die Vermarktung des Körpers und der Genüsse geflochten wird.

Schlüsselwörter: Perversion, Lacan, neoliberale Philosophie 
Citação/Citation: Hoffmann, C. (2014, setembro). Le lien social pervers. Revista Latinoamericana de Psicopatologia Fundamental, 17(3-Suppl.), 666-672.

Editor do artigo/Editor: Prof. Dr. Manoel Tosta Berlinck

Recebido/Received: 15.3.2014/ 3.15.2014 Aceito/Accepted: 17.4.2014 / 4.17.2014

Copyright: (C) 2009 Associação Universitária de Pesquisa em Psicopatologia Fundamental/ University Association for Research in Fundamental Psychopathology. Este é um artigo de livre acesso, que permite uso irrestrito, distribuição e reprodução em qualquer meio, desde que o autor e a fonte sejam citados / This is an open-access article, which permits unrestricted use, distribution, and reproduction in any medium, provided the original author and source are credited.

Financiamento/Funding: $\mathrm{O}$ autor declara não ter sido financiado ou apoiado / The author have no support or funding to report.

Conflito de interesses/Conflict of interest: $\mathrm{O}$ autor declara que não há conflito de interesses

/ The author has no conflict of interest to declare.

\section{Christian Hoffmann}

Psicanalista; Professor na Universidade de Paris VII (Paris, França).

3, rue des Chantiers

75005 Paris, France

e-mail: hoffmann.ch@wanadoo.fr 